\title{
Element Analysis Based on Energy-Dispersive X-Ray Fluorescence
}

\author{
Min Yao, Dongyue Wang, and Min Zhao \\ College of Automation Engineering, Nanjing University of Aeronautics and Astronautics, 29 Yudao Street, Nanjing 210016, China \\ Correspondence should be addressed to Min Yao; ym_nuaa@163.com
}

Received 30 August 2015; Revised 13 November 2015; Accepted 16 November 2015

Academic Editor: Santiago Garcia-Granda

Copyright (C) 2015 Min Yao et al. This is an open access article distributed under the Creative Commons Attribution License, which permits unrestricted use, distribution, and reproduction in any medium, provided the original work is properly cited.

\begin{abstract}
Energy-dispersive X-ray fluorescence (EDXRF) spectrometry is a nondestructive, rapid, multielement, highly accurate, and environment friendly analysis compared with other elemental detection methods. Thus, EDXRF spectrometry is applicable for production quality control, ecological environment monitoring, geological surveying, food inspection, and heritage analysis, among others. A hardware platform for the EDXRF spectrometer is designed in this study based on the theoretical analysis of energy-dispersive X-ray. The platform includes a power supply subsystem, an optical subsystem, a control subsystem, and a personal computer. A fluorescence spectrum analytical method is then developed to obtain the category and content of elements in a sample. This method includes qualitative and quantitative analyses. Finally, a series of experiments is performed. Results show that the precision of the proposed measurement method is below $8 \%$, whereas its repeatability is below $2 \%$.
\end{abstract}

\section{Introduction}

Energy-dispersive X-ray fluorescence (EDXRF) spectrometry exhibits several advantages over other methods that measure elemental content. This technique can measure numerous sample forms and can satisfy different measurement requirements [1]. Moreover, EDXRF spectrometry can detect a wide range of elements, even several elements simultaneously. This technique is nondestructive, fast, highly accurate, and environment friendly. EDXRF spectrometry can be used on different types of sample, such as bulk, liquid, powder, and gas. It can also detect particles in the air.

The European Union enforced a rule called "Restriction of Hazardous Substances" on July 1, 2006, to eliminate several harmful substances in electrical and electronic products. EDXRF spectrometry can rapidly measure these harmful elements in products.

This study introduces the theoretical analysis of energydispersive X-ray, the internal structure of the EDXRF spectrometer, and the algorithm for the rapid analysis of multiple elements. This EDXRF equipment can measure minor elements in materials with contents below $0.03 \%$.

\section{EDXRF Principle}

Direct excitation is a process by which atoms in a specimen are excited by primary photons from external sources, such as an X-ray tube, radioactive source, and synchrotron beam, to produce primary fluorescence. An alternative process is indirect excitation, in which the observed fluorescence is produced as a secondary process by photons or particles (electrons) originating from direct excitation or other secondary processes within the specimen. X-ray is an electromagnetic radiation generated by high-energy particles bombarding atoms. This radiation has wave-particle duality. $\mathrm{X}$-ray fluorescence (XRF) spectrometry uses primary X-ray photons or other microscopic particles to excite the atoms in the test material to produce secondary XRF for material composition analysis and chemical state research. Qualitative analysis of X-ray spectroscopy is based on Moseley's law, and the energy equation is as follows:

$$
E_{\mathrm{X}}=R h C(Z-\sigma)^{2}\left(\frac{1}{n_{1}^{2}}-\frac{1}{n_{2}^{2}}\right),
$$

where $E_{\mathrm{X}}$ is the characteristic X-ray energy, $R$ is Rydberg constant $\left(R_{\infty}=1.09737 \times 10^{7} \mathrm{~m}^{-1}\right), h$ is Planck's constant 


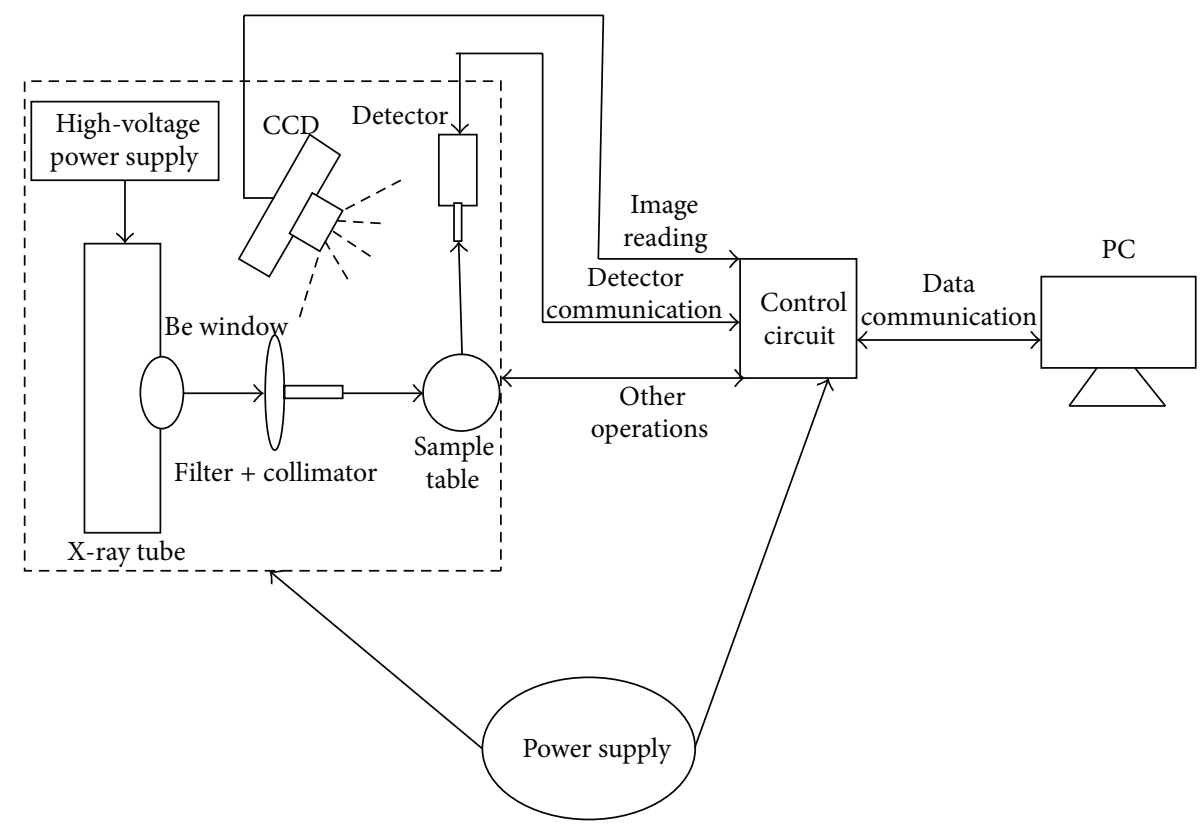

FIGURE 1: Structural diagram of the EDXRF spectrometer.

( $h=6.6262 \times 10^{-34} \mathrm{~J} \cdot \mathrm{s}$ ), and $C$ is the speed of photons. $Z$ is the atomic number, $\sigma$ is Shielding constant, and $n_{1}$ and $n_{2}$ are the energy series. For the spectrum $K \alpha_{1}$, shielding constant $\sigma=1, n_{1}=1$ (K-shell), and $n_{2}=2$ ( $L$-shell). Thus, (1) can be rewritten as follows:

$$
E_{K \alpha_{1}}=\frac{3 R h C(Z-1)^{2}}{4} .
$$

This law reveals the relationship between the X-ray energy and atomic number. This law is the theoretical basis for the qualitative analysis of material composition using XRF. A positive relationship exists between the count rate of the characteristic X-ray and the content of an element of the tested sample, as follows:

$$
I_{K}=\frac{K I_{0}}{\mu_{0}+\mu_{K}} \times W_{K},
$$

where $I_{K}$ and $I_{0}$ are the $K$ layer characteristics of the X-ray of the measured elements and the count rates of the incident $\mathrm{X}$ ray, respectively. Moreover, $\mu_{0}$ and $\mu_{K}$ are the absorption coefficients of the tested substance to the incident X-ray and the tested element to the layer $K$ characteristic X-ray, respectively. $K$ is the constant related to the specific measurement device and should be determined by the calibration instrument $K I_{0} /\left(\mu_{0}+\mu_{K}\right) . W_{K}$ is the measure of the content elements. Quantitative analysis of the measured elements using XRF is theoretically based on (3).

\section{XRF Spectrometer Hardware}

3.1. Structure of the EDXRF Spectrometer. The EDXRF spectrometer is designed according to Moseley's law. The system is illustrated in Figure 1. The spectrometer consists of a power supply, a light path subsystem, a control circuit, and a personal computer (PC). High-voltage power is supplied to the $\mathrm{X}$-ray tube to emit a primary $\mathrm{X}$-ray, which irradiates the sample. The sample is then stimulated to emit XRF, which is received by an XRF detector. The detector classifies the received photons according to energy and counts the number of photons that correspond to different energy levels. The detector then sends the results to the PC, which completes the qualitative and quantitative analyses.

3.2. Light Path Subsystem. The light path subsystem, which includes the X-ray tube, filter, collimator, detector, and a charge-coupled device (CCD) camera, is shown in Figure 2.

The light path subsystem is responsible for emitting, receiving, and counting the $\mathrm{XRF}$ photons. Its operation is as follows. A high-voltage power supply provides highvoltage energy to the $\mathrm{X}$-ray tube, which is stimulated to emit primary X-ray. The primary X-ray passes through the Be window, filter, and collimator, finally irradiating the sample. The sample is stimulated to emit XRF that can be recognized by the detector. The received XRF is transformed into a low-voltage pulse by the preamplifier. The pulse amplitude that is strictly proportional to the energy of the received $\mathrm{XRF}$ is further amplified by the main amplifier. The analogto-digital converter then transforms the amplified voltage into a digital signal. The digital signal is further shaped, sorted, and transformed into a pulse counter with amplitude information. This information is stored in a multichannel analyzer according to its amplitude and finally formatted to an XRF spectral line. The detector transmits spectral information to the PC through a USB hub in the control circuit for qualitative and quantitative analyses. 


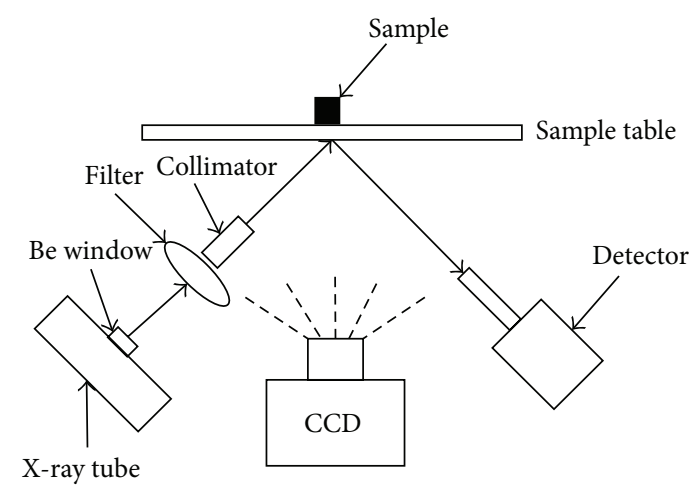

FiguRE 2: Structural diagram of the light path subsystem.

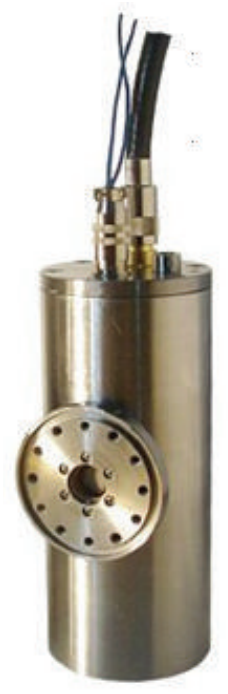

FIGURE 3: XY-501 X-ray tube.

The X-ray tube in the light path subsystem (Model XY501; Dandong Oriental Electron Tube Factory, China) is shown in Figure 3.

This X-ray tube can work at high voltage $(50 \mathrm{kV})$ and low current $(1 \mu \mathrm{A})$. It can also work at low voltage $(4 \mathrm{kV})$ and high current (mA). This tube possesses good stability, keeping low errors of $0.4 \%$ for $4 \mathrm{~h}$. It has a small focal spot size, which is only $0.4 \mathrm{~mm} \times 0.4 \mathrm{~mm}$ with a large $\mathrm{X}$-ray flux.

The detector (Model X-123; Amptek Company, USA) is the key part in the light path subsystem. This part mostly decides the performance of the EDXRF spectrometer. The detector, which is actually a sensor, transforms photons to electrical pulse. The amplitude and number of electrical pluses are related to the energy and intensity of X-ray.

The high-performance detector is shown in Figure 4. The key parameters are listed in Table 1.

The entire measurement is observed using a CCD camera and sent to the PC. The control circuit is responsible for reading or setting the parameters of the tube pressure, switching the filter and the collimator, controlling the interlock, detecting motor position, receiving instructions from the PC, and controlling USB communication.
TABLE 1: X-123 key parameters.

\begin{tabular}{lc}
\hline $\begin{array}{l}\text { Energy resolution }\left(\mathrm{Mn} K_{\alpha}(5.9 \mathrm{eV}) \text { at half }\right. \\
\text { peak height })\end{array}$ & $145 \mathrm{eV}$ \\
\hline Energy & $1.5-25 \mathrm{keV}$ \\
\hline MCA channel number & 2048 \\
\hline Detection area & $5 \mathrm{~mm}^{2}-25 \mathrm{~mm}^{2}$ \\
\hline Counting rate & $2 \times 10^{5} \mathrm{cps}$ \\
\hline
\end{tabular}

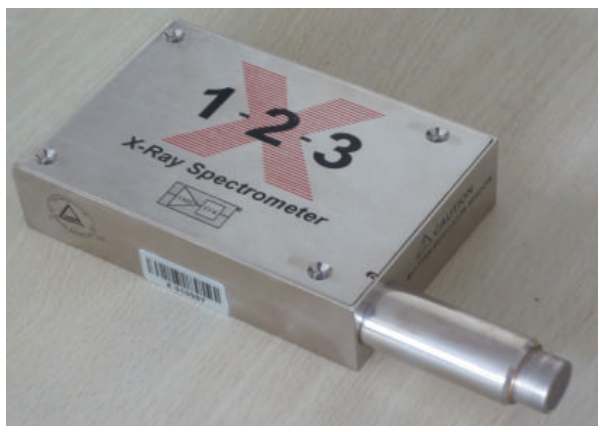

Figure 4: X-123 detector.

\section{XRF Spectral Software}

Qualitative analysis is performed to identify the elements in the sample. By contrast, quantitative analysis is used not only to identify the elements but also to determine elemental content. According to Moseley's law, the XRF photons of a certain element possess a fixed energy; that is, the XRF photons of elements are unique. The abscissa of a channel address reflects the photon energy in the spectral line. The peak position of the abscissa corresponds to the energy of the characteristic XRF of a certain element. Channel address range of a detector in this system is designed from 0 to 2048. Each XRF photon energy ranges from $10^{3} \mathrm{eV}$ to $10^{4} \mathrm{eV}$. Therefore, the enlargement factor of the energy/channel address is designed as $20 \mathrm{eV} /$ channel. For example, the photon energy of $K_{\alpha}$ for $\mathrm{Ag}$ is approximately $22.162 \mathrm{keV}$. The channel address should be near 1108 according to the energy calibration of $20 \mathrm{eV} /$ channel. The $K_{\alpha}$ ray of Ag frequently appears in the channel address of 1106 .

4.1. Qualitative Analysis. Qualitative analysis is the basis of quantitative analysis. Existing element types can be determined using the former analysis. Qualitative analysis is generally divided into three steps.

(1) Peak Location. The uncertainty of a large peak is approximately $\pm 10 \mathrm{keV}$, whereas that of a small peak is up to $\pm 50 \mathrm{eV}$. Small peaks can be neglected when they overlap with a large peak, particularly when its energy level is below $12 \mathrm{keV}$. Spectral overlap and interference frequently occur in the $K$ line (where the atomic number is between 22 and 35) and the $L$ line (where the atomic number is between 56 and 96). 
TABLE 2: Information of the samples.

\begin{tabular}{lcc}
\hline Name & Elemental concentration \% & Manufacturer \\
\hline Cast iron (QD 2021A) & $\mathrm{C}(3.57), \mathrm{S} \mathrm{(0.053)}$ & East Institute of Standard Material, Jinan (2012) \\
High-Mn steel (QD 98-22) & $\mathrm{C} \mathrm{(0.68),} \mathrm{Si}(0.254)$ & East Institute of Standard Material, Jinan (2010) \\
Lead-based alloy (Pb60 Sn40) & $\mathrm{Pb}(59.02), \mathrm{Sn}(40.54)$ & Shandong Research Institute of Metallurgical Science (1995) \\
Brass (GBW(E) 020012) & $\mathrm{Cu} \mathrm{(63.34),} \mathrm{Pb}(0.028)$ & Shanghai Institute of Electrical and Mechanical Engineering (2002) \\
\hline
\end{tabular}

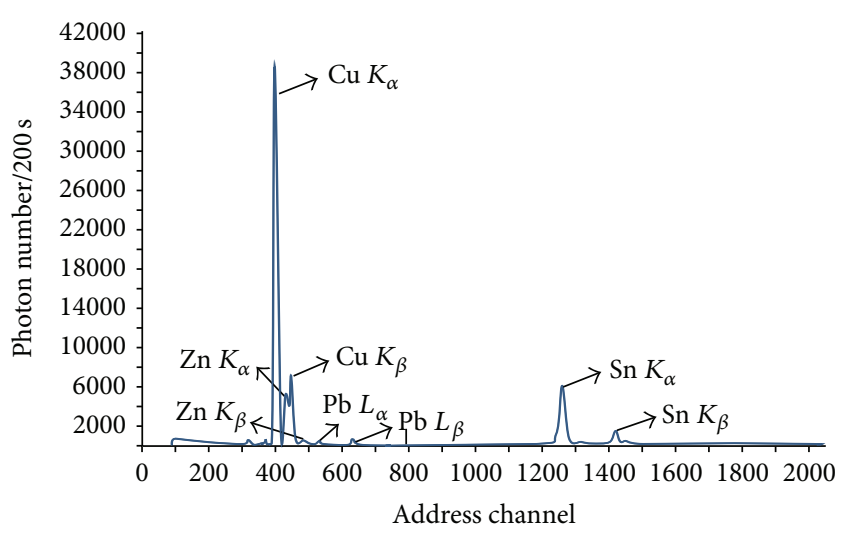

Figure 5: Spectral line of $\mathrm{Cu}$.

All aforementioned factors hamper an accurate qualitative analysis.

(2) Peak Recognition. The uncertainty of the peak position generally increases the difficulty of peak recognition. More than one peak corresponds to the energy peak in the spectrum in several cases. Accumulated, escape, and scattering peaks can also interfere with recognition.

(3) Element Determination. Except for light elements, such as $\mathrm{Na}, \mathrm{Mg}, \mathrm{Al}, \mathrm{Si}, \mathrm{P}$, and $\mathrm{S}$, identifying an element typically requires more than two characteristic spectral peaks. When the voltage of the $\mathrm{X}$-ray tube is over $30 \mathrm{keV}$ for atomic numbers ${ }^{19} Z$ to ${ }^{42} Z$, the spectral peaks $K_{\alpha}$ and $K_{\beta}$ appear simultaneously. Furthermore, relative intensities in different spectral peaks should also be considered [2].

Figure 5 is the spectral line of $\mathrm{Cu}$ when the tube voltage is $40 \mathrm{keV}$, tube flow is $600 \mu \mathrm{A}$, and the optical filter is $800 \mu \mathrm{m}$.

The characteristic peak area of the relative element in the qualitative analysis is calculated. This area corresponds to the photon number of the relative energy. The intensity of the characteristic peak can then be achieved. However, interference peaks should be considered in calculating the peak area.

The peak in the peak location, which is an interference peak to the main peak, is called a pseudopeak. Such peak includes accumulated, escape, and scattering peaks. The accumulated peak is also called the sum peak, which is a phenomenon of peak hyperplasia resulting from the accumulation of signal pulses while counting at a high rate. In a sum peak, the peak position does not correspond to the characteristic energy but to the sum of two independent peaks [2].
When the energy of an X-ray photon is higher than the detection limit, some energy of the characteristic X-ray can escape because of its high transparency. The escaped energy forms an escape peak in the low-energy position. The energy difference between the main and escape peaks is equal to that of the energy of the characteristic X-ray photons, which is recognized by the detector. The Si-PIN detector shows that the escape peak energy is $1.74 \mathrm{keV}$ lower than the main peak, which is the Si $K_{\alpha}$ energy. The height of the escape peak is approximately $1 / 1000$ to $2 / 100$ of the main peak. However, the escape peak does not appear when the atomic number is over 30 [3].

X-ray scatters when it passes through objects, and a scattering peak appears. Two types of scattering peaks are observed, namely, coherent scattering (or Rayleigh scattering) and incoherent scattering (or Compton scattering). The scattering peak in the EDXRF spectrometer mainly results from the stimulation of the anode target of the X-ray tube.

4.2. Quantitative Analysis. Quantitative analysis depends on the standard curve established by standard samples. The measured intensity value of the unknown element in an actual measurement is fed into the standard curve to obtain the elemental contents. In particular, the standard samples and unknown element should be measured under similar conditions.

Detailed steps are illustrated in Figure 6.

\section{Experiments}

The EDXRF spectrometer is shown in Figure 7. The sample table in Figure 7(c) holds the sample. Figure 7(d) shows the control circuit that controls the instrument and communicates with the PC.

Four types of samples are used in the tests. Sample characteristics are listed in Table 2.

The EDXRF spectrometer follows a set of operating steps, namely, preheating, initialization, mode selection, measurement starting, qualitative analysis, and quantitative analysis.

The EDXRF spectrometer should be preheated for approximately $40 \mathrm{~min}$ before the experiment. Preheating is performed to increase tube pressure and tube flow gradually to protect the light pipe and stabilize the instrument more.

Initialization is necessary to calibrate channel magnification. A standard Ag sample is stimulated and magnification is adjusted during initialization. This step does not terminate until the main characteristic XRF stimulated from $\mathrm{Ag}$ is located near the address of 1106. This step can ensure that system hardware remains in the best situation. Figure 8 shows 


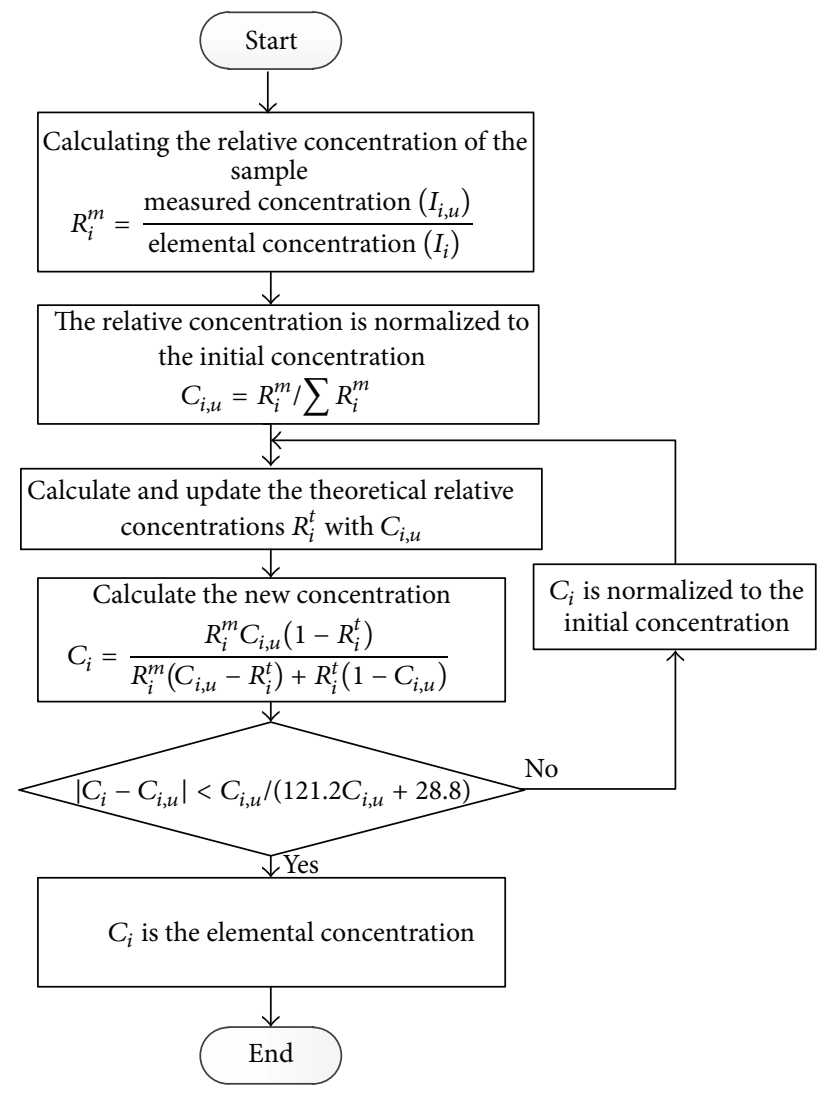

FIGURE 6: Steps of quantitative analysis, where $m$ is the measured value and $t$ is the calculated value.

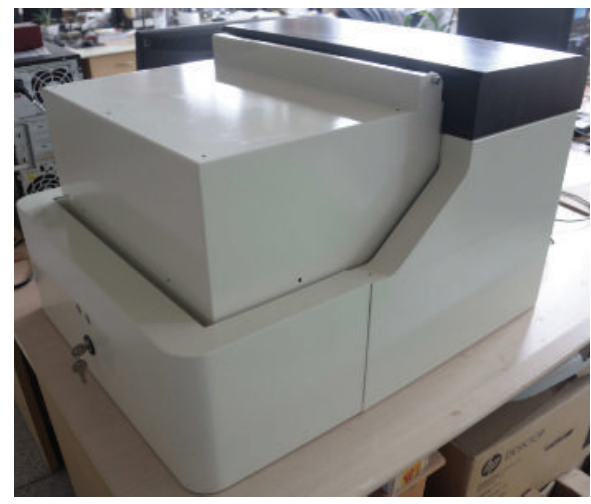

(a) Instrument appearance

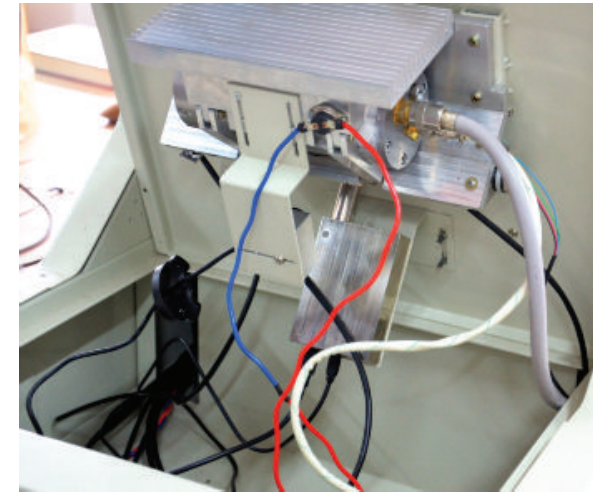

(b) Light path subsystem

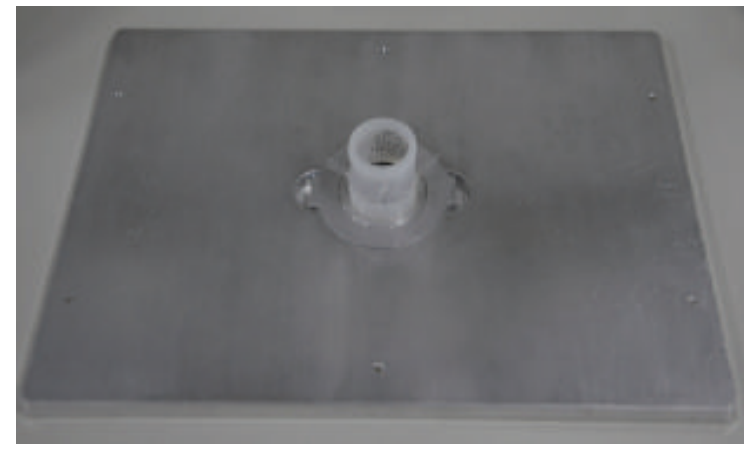

(c) Sample table

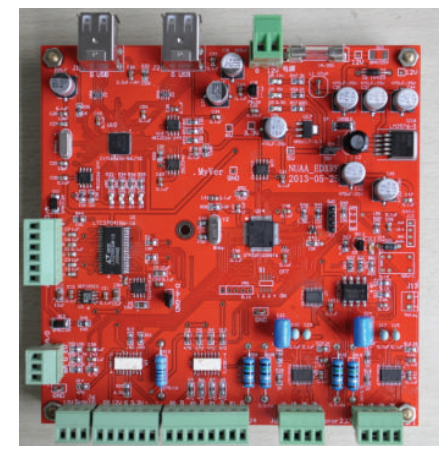

(d) Control circuit

FIGURE 7: EDXRF spectrometer. 


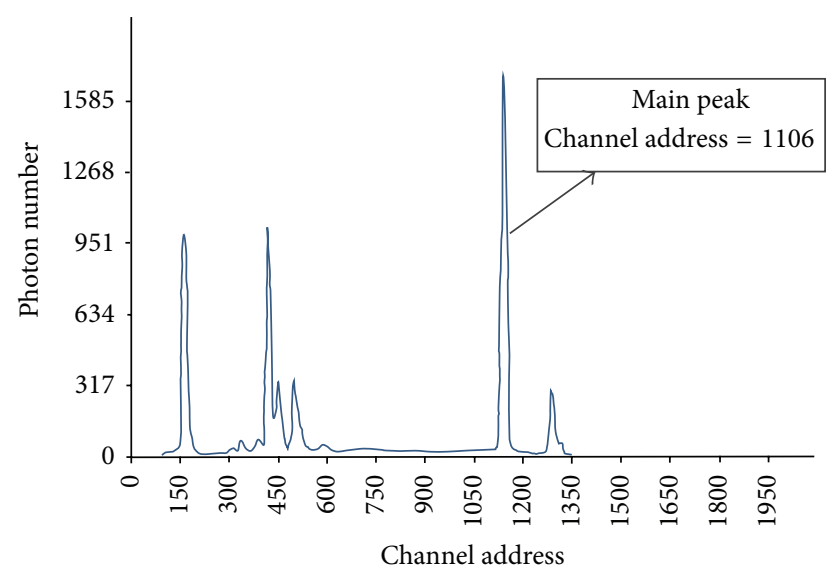

FIgURE 8: System initialization.

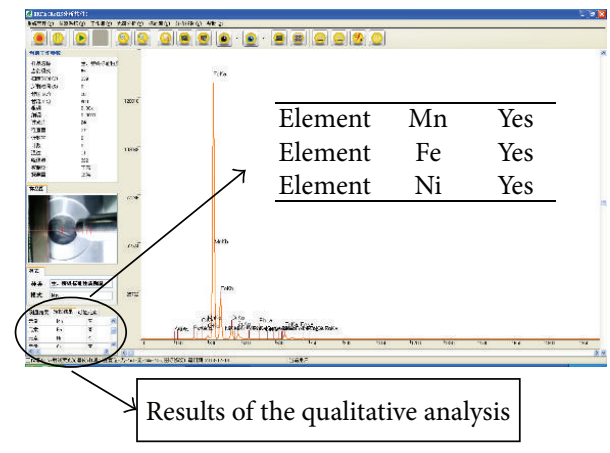

Figure 9: Results of the qualitative analysis.

the spectral line of Ag when the system has been completely initialized.

Measurement is started after the suitable working mode has been established. An experiment is performed to measure the Mn content of cast iron sample. Figure 9 presents the results of the qualitative analysis, which show elements $\mathrm{Mn}$, $\mathrm{Fe}$, and $\mathrm{Ni}$ in the cast iron. Meanwhile, Figure 10 presents the results of the quantitative analysis, which show that $\mathrm{Mn}$ content is $12,335 \mathrm{ppm}$ or $1.23 \%$.

The experiments are conducted 15 times to calculate the repeatability of the results. Experimental results are presented in Table 3.

Another experiment is conducted to measure the Mn content of high-Mn steel. The results are presented in Table 4.

The relative error for the Mn content of cast iron is within $5 \%$, whereas that for Mn content of high-Mn steel is within $3 \%$. Repeatability of the results is calculated, and the results are presented in Table 5.

Table 3 shows that the repeatability of the measurement is within $2 \%$.

Two more experiments are conducted to measure minor elements. The results are shown in Tables 6 and 7.

The results from the two experiments show that the proposed EDXRF method can easily measure minor elements in materials.

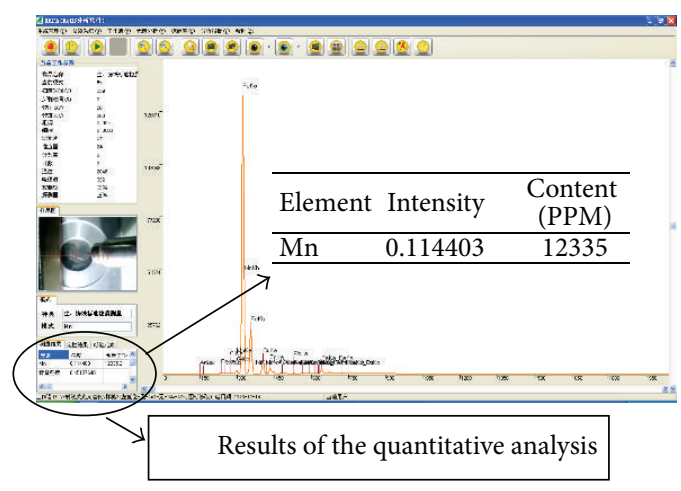

FigURE 10: Results of the quantitative analysis.

TABLE 3: Mn content of cast iron.

\begin{tabular}{|c|c|c|c|}
\hline \multirow{2}{*}{ Number } & \multicolumn{3}{|c|}{$\mathrm{Mn}$} \\
\hline & Measured value & Reference value & Relative error \\
\hline 1 & $1.32 \%$ & & $4.76 \%$ \\
\hline 2 & $1.29 \%$ & & $2.38 \%$ \\
\hline 3 & $1.20 \%$ & & $-4.76 \%$ \\
\hline 4 & $1.24 \%$ & & $-1.58 \%$ \\
\hline 5 & $1.23 \%$ & & $-2.38 \%$ \\
\hline 6 & $1.27 \%$ & & $0.79 \%$ \\
\hline 7 & $1.28 \%$ & & $1.58 \%$ \\
\hline 8 & $1.22 \%$ & $1.26 \%$ & $-3.17 \%$ \\
\hline 9 & $1.25 \%$ & & $-0.79 \%$ \\
\hline 10 & $1.20 \%$ & & $-4.76 \%$ \\
\hline 11 & $1.21 \%$ & & $-3.97 \%$ \\
\hline 12 & $1.22 \%$ & & $-3.17 \%$ \\
\hline 13 & $1.32 \%$ & & $4.76 \%$ \\
\hline 14 & $1.23 \%$ & & $-2.38 \%$ \\
\hline 15 & $1.22 \%$ & & $-3.17 \%$ \\
\hline
\end{tabular}

TABLE 4: Mn content of high-Mn steel.

\begin{tabular}{|c|c|c|c|}
\hline \multirow{2}{*}{ Number } & \multicolumn{3}{|c|}{$\mathrm{Mn}$} \\
\hline & Measured value & Reference value & Relative error \\
\hline 1 & $12.10 \%$ & & $-0.33 \%$ \\
\hline 2 & $12.12 \%$ & & $-0.16 \%$ \\
\hline 3 & $12.14 \%$ & & $0 \%$ \\
\hline 4 & $11.92 \%$ & & $-1.81 \%$ \\
\hline 5 & $12.49 \%$ & & $2.88 \%$ \\
\hline 6 & $12.41 \%$ & & $2.22 \%$ \\
\hline 7 & $12.21 \%$ & & $0.57 \%$ \\
\hline 8 & $12.13 \%$ & $12.14 \%$ & $-0.08 \%$ \\
\hline 9 & $12.14 \%$ & & $0 \%$ \\
\hline 10 & $11.91 \%$ & & $-1.89 \%$ \\
\hline 11 & $12.07 \%$ & & $-0.57 \%$ \\
\hline 12 & $12.34 \%$ & & $1.65 \%$ \\
\hline 13 & $12.05 \%$ & & $-0.74 \%$ \\
\hline 14 & $12.03 \%$ & & $-0.91 \%$ \\
\hline 15 & $12.35 \%$ & & $1.73 \%$ \\
\hline
\end{tabular}


TABLE 5: Repeatability of the measurement results.

\begin{tabular}{lcc}
\hline Element & Content & Repeatability \\
\hline \multirow{2}{*}{$\mathrm{Mn}$} & $1.26 \%$ & $0.08 \%$ \\
& $12.14 \%$ & $1.56 \%$ \\
\hline
\end{tabular}

TABLE 6: Cu content of lead-based alloy.

\begin{tabular}{|c|c|c|c|}
\hline \multirow{2}{*}{ Number } & \multicolumn{3}{|c|}{$\mathrm{Cu}$} \\
\hline & Measured value & Reference value & Relative error \\
\hline 1 & $0.0268 \%$ & \multirow{10}{*}{$0.0270 \%$} & $-0.74 \%$ \\
\hline 2 & $0.0267 \%$ & & $-1.11 \%$ \\
\hline 3 & $0.0271 \%$ & & $0.37 \%$ \\
\hline 4 & $0.0268 \%$ & & $-0.74 \%$ \\
\hline 5 & $0.0271 \%$ & & $0.37 \%$ \\
\hline 6 & $0.0272 \%$ & & $0.74 \%$ \\
\hline 7 & $0.0271 \%$ & & $0.37 \%$ \\
\hline 8 & $0.0270 \%$ & & 0 \\
\hline 9 & $0.0273 \%$ & & $1.11 \%$ \\
\hline 10 & $0.0271 \%$ & & $0.37 \%$ \\
\hline
\end{tabular}

TABLE 7: Cu content of brass.

\begin{tabular}{lccc}
\hline Number & \multicolumn{3}{c}{ cu } \\
& Measured value & Reference value & Relative error \\
\hline 1 & $0.0850 \%$ & & $2.18 \%$ \\
2 & $0.0756 \%$ & & $-3.07 \%$ \\
3 & $0.0831 \%$ & & $6.54 \%$ \\
4 & $0.0841 \%$ & & $7.82 \%$ \\
5 & $0.0743 \%$ & & $-4.74 \%$ \\
6 & $0.0729 \%$ & $0.0780 \%$ & $-6.54 \%$ \\
7 & $0.0727 \%$ & & $-6.79 \%$ \\
8 & $0.0833 \%$ & & $6.79 \%$ \\
9 & $0.0718 \%$ & & $-7.95 \%$ \\
10 & $0.0719 \%$ & & $-7.82 \%$ \\
\hline
\end{tabular}

\section{Conclusion}

XRF analytical method is discussed according to Moseley's law. An EDXRF spectrometer, which consists of a power supply, a light path subsystem, and a control circuit, is designed. A series of experiments is conducted using this instrument. The results show that the precision of this equipment for measuring elemental content is below $8 \%$, and its repeatability is below $2 \%$.

\section{Conflict of Interests}

The authors declare that there is no conflict of interests regarding the publication of this paper.

\section{Acknowledgment}

This research has been funded by Nanjing University of Aeronautics and Astronautics Research Funding (Grant no. NS2015030).

\section{References}

[1] T. Wakisaka, N. Morita, M. Wakasa, S. Terada, K. Nishihagi, and K. Taniguchi, "Development of energy dispersive X-ray fluorescence spectrometer with monochromatic excitation for the direct determination of trace elements in organic matrices," Bunseki Kagaku, vol. 45, no. 10, pp. 933-939, 1996.

[2] A. Gürol, "Measurements of the K X-ray intensity ratios by using energy-dispersive X-ray fluorescence spectrometry," Applied Radiation and Isotopes, vol. 66, no. 3, pp. 372-376, 2008.

[3] R. Sitko, B. Zawisza, and E. Malicka, "Energy-dispersive Xray fluorescence spectrometer for analysis of conventional and micro-samples: preliminary assessment," Spectrochimica Acta Part B: Atomic Spectroscopy, vol. 64, no. 5, pp. 436-441, 2009. 

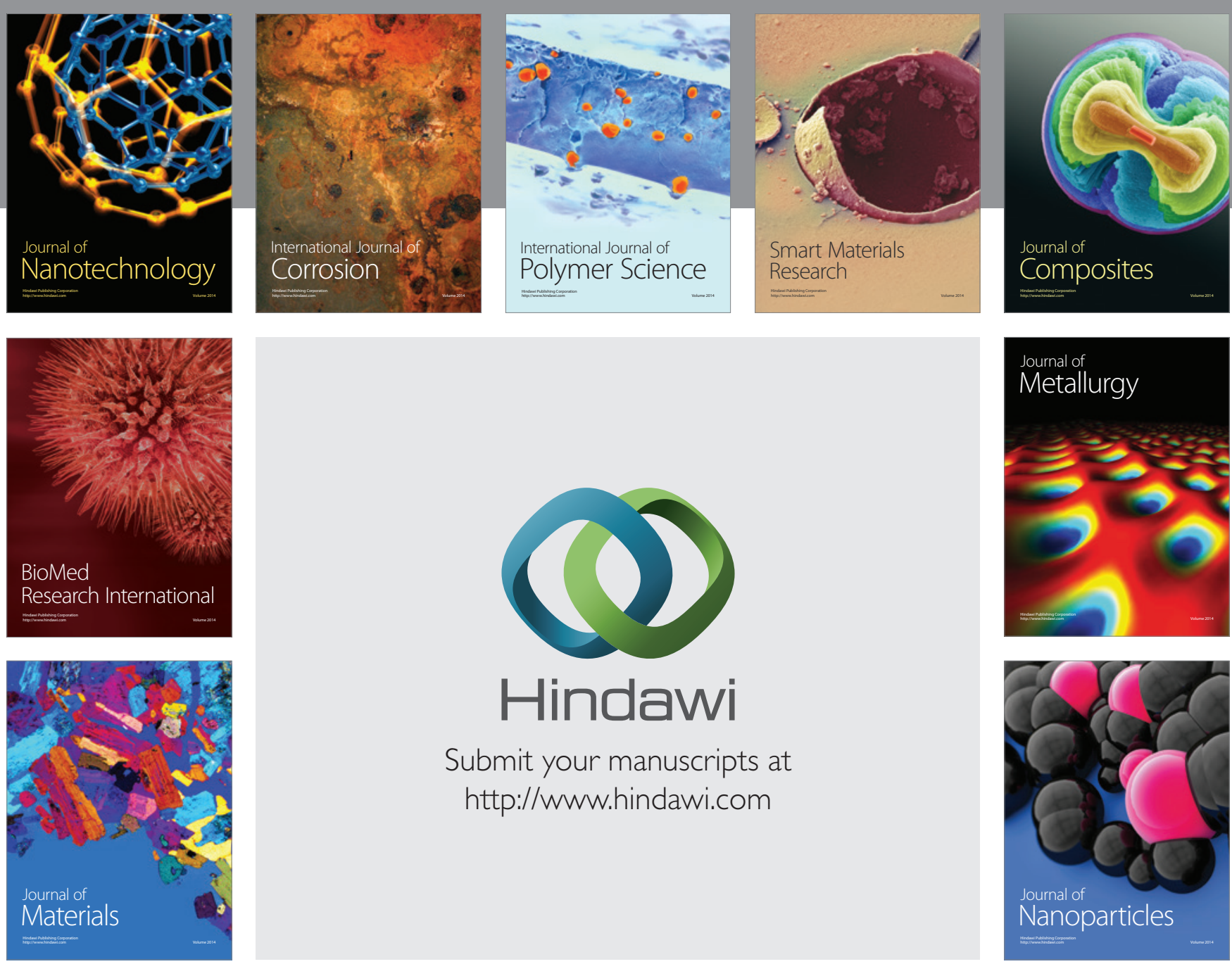

Submit your manuscripts at http://www.hindawi.com
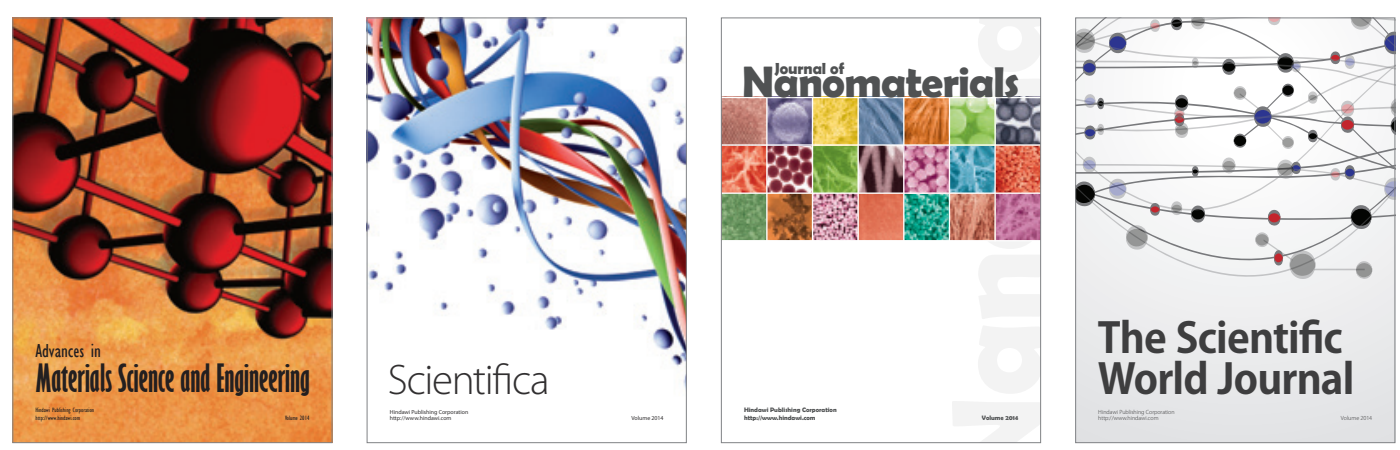

\section{The Scientific World Journal}
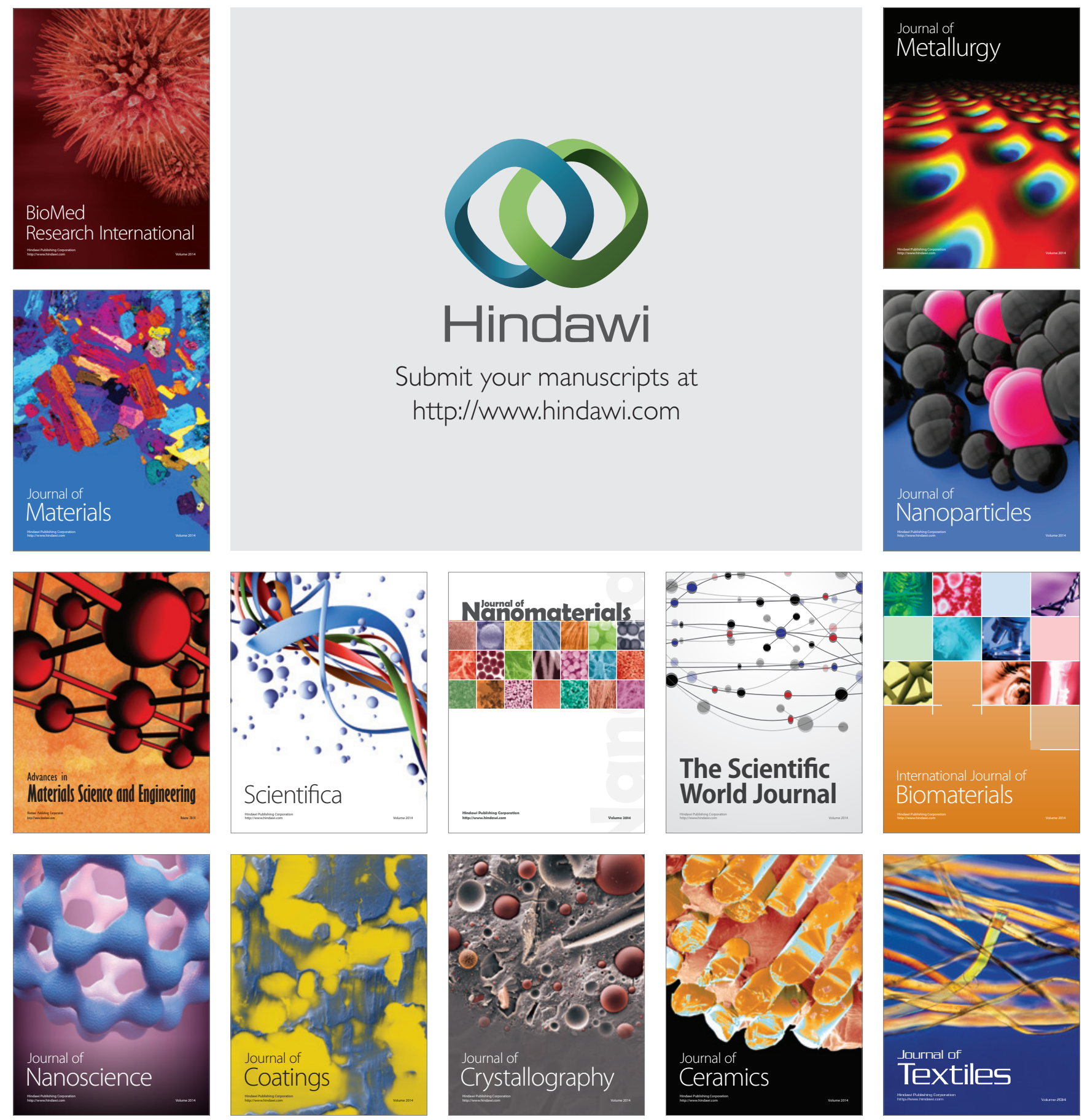